\title{
CONCEPCIÓN DIDÁCTICA PARA LA DISCIPLINA CONTABILIDAD EN EL CICLO BÁSICO DE LA CARRERA CONTABILIDAD Y FINANZAS DE LA UNIVERSIDAD DE PINAR DEL RÍO
}

\author{
DIDACTIC CONCEPTION FOR DISCIPLINE ACCOUNTING IN THE \\ BASIC CYCLE OF THE CAREER ACCOUNTING AND FINANCES OF \\ THE UNIVERSITY OF PINAR DEL RÍO
}

\author{
Reinaldo Castaño de Armas \\ Juan Carlos Díaz Cabrera \\ Jorge Luis Mena Lorenzo
}

Universidad Pinar del Río. Calle Martí 270 final. Cuba. riny@upr.edu.cu

\section{RESUMEN}

La investigación está dirigida a proponer una concepción didáctica para el proceso de enseñanzaaprendizaje durante el ciclo básico para los estudiantes de primero y segundo año de la carrera de Contabilidad y Finanzas de la Universidad de Pinar del Río, para ello se fundamentó una concepción didáctica sustentada en las relaciones interdisciplinarias, donde la disciplina de Contabilidad direcciona el proceso de integración horizontal y vertical, a partir de la simulación de una empresa, que permite modelar los subsistemas del sistema contable, apoyado por un sistema de medios de enseñanza que organizan y direccionan el proceso de aprendizaje. Los métodos empleados fueron: histórico y lógico, análisis y síntesis, inducción-deducción, sistémico estructural, observación, revisión de documentos, encuestas, entrevistas, los métodos estadísticos y matemáticos. Se presenta una metodología con el objetivo de implementar una concepción didáctica que permita a los profesores que intervienen en el proceso de formación del contador, utilizando los recursos metodológicos y didáctico para perfeccionar el proceso de enseñanza-aprendizaje haciendo énfasis en la impartición de talleres en la disciplina de Contabilidad, Diplomado de Actualización de los cambios en la Contabilidad a partir de la armonización de las Normas Cubanas de Contabilidad a las Normas Internacionales y de Información Financiera.

Palabras Claves: Integración, interdisciplinarias, horizontal, vertical, virtual, modelar, subsistema.

\begin{abstract}
This investigation is guided to make perfect the Accounting teaching-learning process during the basic cycle for Accounting and Finances first and second year students at Pinar del Río University. For this purpose it was given a didactic conception sustained on the interdisciplinary relations where the Accounting discipline directs the horizontal and vertical integration process, starting from a virtual company's simulation that allows modeling the countable system's subsystems, based on a teaching means system that organize and direct the teaching-learning process. The employed methods were: historic and logic, analysis and synthesis, induction - deduction, systemic structural, observation, documents revision, inquiry, interview, the statistical methods and mathematicians. As a practical contribution, it is offered a methodology to implement the didactic conception that provides to the professors that intervene in the counter formation process, the methodological and didactic resources to make perfect the teaching- learning process doing emphasis on the workshops impartation in the Accounting discipline and in an Actualization's Diploma about the Accounting changes starting from the harmonizing of Cuban Accounting's Standards to the Internationals' Standards and Financial Information.
\end{abstract}

Keywords: integration, interdisciplinary, horizontal, vertical, virtual, modeling, subsystem.

Recibido: 29/10/2017;

Aceptado: 04/12/2017;

Publicado: 19/12/2017

\section{Introducción}

En el desarrollo histórico de la humanidad, las universidades han jugado un papel estratégico en la formación de profesionales capaces de solucionar problemas, accediendo al conocimiento científico. El siglo XXI depara de la Educación Superior nuevos retos, que deben enfrentar con creatividad y criterio innovador. La situación de la globalización de la cultura y 
el patrimonio en un contexto de apertura de las economías, la insuficiencia de recursos para el sector, el nuevo rol de la ciencia en la producción, el acceso y la utilización de la información, entre otros, son una muestra clara de la magnitud del desafío.

En Cuba, la solución más generalizada ante esta situación ha sido la formación integral del profesional, mediante el incremento de su preparación básica y el empleo de métodos de enseñanza que desarrollen el pensamiento creativo de los egresados y sus posibilidades de adaptación a nuevas tendencias en el mundo globalizado. (1) (Tristá, 1985:12).

En las condiciones macroeconómicas y financieras que la economía cubana se inserta a los mercados internacionales, la estrategia económica que ha desarrollado durante estos difíciles años que la vida ha confirmado como certera. Exige la formación de un profesional capaz de interpretar y asimilar los avances tecnológicos que en lo fundamental se desarrollan bajo la fuerte presión que ejerce el proceso de perfeccionamiento de la Educación Superior y en particular de la carrera de Contabilidad y Finanzas.

Sin embargo, en la práctica educativa de las ciencias contables para esta carrera en la Universidad de Pinar del Río, la situación no muestra una mejoría sustancial. Así se refleja en los resultados de la exploración realizada que incluyó el análisis de informes de visitas de inspección del Ministerio de Educación Superior (MES) a la carrera, el estudio del reglamento para el Trabajo Docente y Metodológico en la Educación Superior del año 2007, el estudio de los programas de las asignaturas de esta disciplina incluyendo la Disciplina Principal Integradora de primer año y segundo. $Y$ además se utilizaron como instrumento las entrevistas, encuestas aplicadas a profesores, directivos de la carrera y estudiantes.

Por lo tanto se constatan las siguientes limitaciones:

$\checkmark$ El proceso de enseñanza-aprendizaje se convierte en un acto academicista, que incluye pobremente el modelo de empresa cubana como formación temprana del pensamiento lógico del profesional.

$\checkmark$ La enseñanza se desarrolla con metodologías tradicionales que tienden a limitar el aprendizaje de contenidos con significatividad y sentido personal.

$\checkmark$ Los procesos sustantivos (académicolaboral-investigativo) carecen de integración entre las asignaturas del ciclo y favorecen poco la horizontalidad y verticalidad de la enseñanza-aprendizaje.

Todo lo anterior expuesto ha influido en que los estudiantes presentan limitaciones para el tránsito por la carrera, manifestando bajos niveles de aprendizaje en cuanto a la identificación de procesos y hechos contables así como la formación de un lenguaje técnico que les permita comunicarse con fluidez en el entorno empresarial.

La lenta adaptación al mundo laboral, tan complejo a partir de las relaciones monetarias mercantiles que se establecen, por la implementación del nuevo modelo económico y la apertura al mercado internacional se han caracterizado por la globalización y el desarrollo vertiginoso de las ciencias.

\section{Objetivo}

Desde los antecedentes expuesto los autores se cuestionan ¿Cómo perfeccionar el proceso de enseñanza-aprendizaje de la disciplina Contabilidad durante el ciclo básico de la carrera de Contabilidad y Finanzas de la Universidad de Pinar del Río?, a partir de esta interrogante profundizan en el estudio del proceso de enseñanza-aprendizaje de la disciplina de Contabilidad durante el ciclo básico en la carrera de Contabilidad y Finanzas de la Universidad de Pinar del Río.

La respuesta a esta interrogantes se realiza a través del siguiente objetivo: Proponer una concepción didáctica para el proceso de enseñanza-aprendizaje de la disciplina de Contabilidad durante el ciclo básico de la carrera que permita su implementación a través de una metodología en la Universidad de Pinar del Río en correspondencia con los cambios que se están gestan en el mundo global actual.

\section{Materiales y métodos}

La investigación realizada tiene una fundamentación dialéctico-materialista como método general. Se utilizó en todo el proceso al operar con categorías, leyes y principios como herramientas imprescindibles para los análisis, que permitieron revelar las relaciones existentes, según el tema investigado. Se emplearon métodos de investigación en los niveles teóricos, empíricos y estadísticomatemáticos.

Dentro de los métodos del nivel teórico se encuentran el histórico y lógico, a partir de realizar la evolución de los estudios contables en Cuba, análisis y síntesis, induccióndeducción, sistémico estructural, con los que se 
puede fundamentar el proceso de enseñanzaaprendizaje de la disciplina de Contabilidad durante el ciclo básico de la carrera, para con posterioridad arribar a la concepción didáctica y la metodología para su implementación en la Universidad de Pinar del Río.

Se utilizan además los métodos del nivel empírico como son: observación al proceso docente educativo, revisión de documentos dentro de los que se encuentran los planes y programas de la carrera y disciplina de Contabilidad, encuestas y entrevistas a directivos, profesores y estudiantes de la Universidad de Pinar del Río.

\section{Resultados}

El proceso de enseñanza-aprendizaje de la Contabilidad. Acercamiento a la Evolución Historia de la enseñanza-aprendizaje de la Contabilidad.

La ciencia de la Contabilidad es tan antigua como la necesidad que tiene una persona de disponer de información para cuantificar los beneficios obtenidos de la realización de actividades comerciales. Durante el siglo XV ocurrieron acontecimientos que importaron fuertemente a la técnica contable como para considerarse que en ese periodo se gestaron las bases de lo que se conoce hoy como Contabilidad, efectivamente, en esa época se le atribuyó la «paternidad» de la Contabilidad a un monje llamado Fray Lucas Paccioli (1494). La doctrina contable expuesta por Paccioli en su obra se difundió rápidamente por toda Europa y, principalmente, por los países que habrían de sustituir en la hegemonía económica a las ricas y cultas ciudades italianas.

Durante la crisis de (1929) ocurrió lo que se conoce como la Gran Depresión de los treinta, período caracterizado por una crisis financiera que se originó en los Estados Unidos y tuvo repercusión Mundial. Una de las consecuencias de dicha crisis fue estandarizar, reforzar y redimensionar el rol de la Contabilidad y un reordenamiento de la enseñanza-aprendizaje, caracterizada por el escolasticismo, enseñanza repetitiva y memorística dirigida a sectores pudientes de la sociedad en el poder.

En 1951 se crea la Unión Europea de Expertos Contables (UEEC) y se consolida en (1963), entre sus objetivos: comparar los métodos nacionales de formación profesional y de acceso a la profesión, con vistas a la cooperación europea; no publicó recomendaciones sobre formación, pero sí borradores, en (1981) se transforma en la Federación Europea de Expertos Contables (FEEC). (Moracho, 1915:12).

Al finalizar la segunda guerra mundial, la UNESCO propuso la creación de un Instituto Internacional de Contabilidad, que no vio la luz por las diferencias de nivel y legislación en el seno de la ONU, en plena guerra fría. El segundo intento tiene lugar en el seno de las Naciones Unidas sobre Comercio y Desarrollo (UNCTAD) creada en 1964. (Moracho, 1915:14).

En octubre 1977 se crea la Federación Internacional de Contadores (IFAC), en sus siglas en Inglés, simultáneamente se crea el Comité de Formación, actual International Accounting Education Standard Board (IASB). Entre sus objetivos está el establecer pautas sobre la formación de los profesionales de la contabilidad, 63 miembros/54 países. (Moracho, 1915:19)

En 1978, en su tesis doctoral en autor Aquiles Limone propone un modelo titulado, Proceso Primario en el conjunto secuencial de transformaciones, realizadas en y por la empresa, que tiene por objeto la regeneración de los fondos consumidos en el mantenimiento de su estructura y de los fondos utilizados en esas transformaciones que experimentan los flujos de recursos en el ciclo operacional de una empresa.

En 1983, en la XI Jornada de Ciencias Económicas del ConoSur los autores Carlos Alvarez; María Teresa Garcías y Francisco Nattero proponen una metodología docente para el área contable en la formación del contador, en la que se enfatiza la enseñanza a partir de los procesos primarios y de un principio contable fenomenológico, la Dualidad Económica.

A finales de la década de los 90 , se inició en Estados Unidos un movimiento en apoyo del cambio en la forma de enseñar la contabilidad, encabezado por el Banco Mundial. Así organismos como la American Accounting Association (AAA) o la International Financial Accounting Committe (IFAC), pusieron de manifiesto que la educación en contabilidad ha estado demasiado centrada en la adquisición de habilidades técnicas por parte de los alumnos (Adler y Milne, 1997:45), dando lugar a una enseñanza basada en un enfoque individual y pasivo (Hwang et al., 2005:56). Estos organismos sugieren que en la enseñanza de la Contabilidad existe un déficit en el fomento de otras habilidades y capacidades, tales como la resolución de problemas y las habilidades de comunicación e 
interpersonales. Por ello proponen a los educadores el empleo de métodos basados en casos reales, seminarios, juegos de rol, simulaciones y otras técnicas que envuelven al estudiante activamente en el proceso de aprendizaje (Donoso y López, 2010:12).

A partir de los requisitos establecidos por la UNCTAD y acorde a las particularidades de los países latinoamericanos, La Asociación Latinoamericana de Facultades y Escuelas de Contaduría (ALAFEC) cumpliendo el encargo del Consejo Ejecutivo Ampliado, realizada en México en el mes de marzo del 2002, presentó a la Asamblea General de ALAFEC, en la Ciudad de Mérida, Yucatán, México, realizado en noviembre del 2003 entre otros, el tema central: "Las Bases para la Formación del Contador Público en América Latina".(Konja, 2008:10).

Objetivos propuestos en la definición de las bases para la creación de un plan de estudio latinoamericano para la Contaduría Pública.

Alcanzar una sólida base académica con un alto contenido teórico en todas las áreas del conocimiento definidas en la licenciatura para que permita la homologación del quehacer profesional una vez graduado el estudiante en cualquier país de Latinoamérica.

Tener la suficiente flexibilidad para que cada país o región pueda exponer en el Plan de Estudios sus propias características 0 particularidades adecuadas a la realidad local.

Integrar el sistema de conocimientos y habilidades adquiridos en el Plan de Estudios en el contexto nacional y latinoamericano.

En el 2009, Berta Silva Palavecinos en su artículo" El aprendizaje de los fundamentos contable basado en el enfoque del Proceso Primario". En el modelo de Aquiles Limone, el supuesto de que el ciclo se inicia con determinado monto de efectivo y que el incremento o decremento que se genera en ese nivel de efectivo durante el período corresponde a la variación patrimonial, constituía una limitación en el modelo. Ella consiste en la posibilidad de confundir las variaciones patrimoniales y las variaciones de Caja y de dificultar la comprensión del concepto devengado como base contable que se utiliza y que además constituye un Supuesto Fundamental establecido en el Marco Conceptual de International Accounting Standards Board (IASB).

El proceso de Enseñanza-Aprendizaje de la Contabilidad. Particularidades en Cuba.
El contexto mundial actual, caracterizado por la globalización de la enseñanza, la apertura de la economía cubana a la inversión extranjera a partir de la implementación del nuevo modelo económico, la armonización del sistema contable a estándares internacionales de la información financiera, ha provocado un proceso de profundas transformaciones socioeconómicas, por lo que se convierte en una necesidad el perfeccionamiento del proceso de enseñanza-aprendizaje de la Contabilidad.

Los estudios contables en Cuba se iniciaron 1927 cuando en la Universidad de La Habana se creó la Escuela Superior de Ciencias Comerciales, constituyendo esta la primera Escuela Universitaria Cubana, que tuvo como antecedentes otros estudios de nivel medio en Escuelas de Comercio.

El período de 1959 a 1967 se evidenció un proceso de profundas trasformaciones en los programas de estudio de la carrera de Contabilidad y Finanzas.

El año 1967 marca un momento de cambio absoluto en la concepción de la formación académica del profesional de las Ciencias Contables y Financieras en Cuba derivado de las condiciones que prevalecen en el entorno empresarial cubano. El no reconocimiento de las relaciones monetario mercantiles entre las empresas estatales en la economía trae como consecuencia el establecimiento de un sistema de registro económico que se centraba en la utilización de indicadores materiales, esta nueva concepción deriva en una disminución abrupta del reconocimiento social hacia la profesión del contador. La situación así planteada trae como consecuencia la necesidad de transformar los planes y programas de estudios universitarios variándose inclusive el perfil profesional, surge así una Licenciatura en Control Económico y desaparece la formación de Contadores Públicos.

A partir de la aprobación de las bases para la creación del Plan de estudio para la formación del Contador Público en América Latina por la ALAFLEC, con el objetivo de homologar e integrar el sistema de conocimientos, habilidades y valores en el quehacer profesional de los egresados de esta carrera en cualquier país de Latinoamérica. Este plan de estudios constituyó el punto de partida para la realización del Plan de Estudio "D" de esta carrera en Cuba.

Principales nudos conceptuales: 
La formación de los profesionales en las Ciencias Contables conlleva a un compromiso de las universidades con la sociedad. "Una de las formas de cumplir con este compromiso es llevar a cabo la actualización constante de los currículos académicos, que constituyen la guía o brújula que el profesor debe utilizar y además cumplir en el proceso de enseñanzaaprendizaje de la Contabilidad." (Donoso, 2009:45)

En este sentido, el docente tiene necesidad de modificar, perfeccionar la manera de cómo se desarrolla actualmente el proceso de enseñanza- aprendizaje de la Contabilidad y asumir otras vías, formas, conceptos que proyecten al ejercicio de esta actividad más allá de la técnica, que permita la integración teóricapráctica de los contenidos, así de esta forma activar los procesos lógicos del pensamiento durante el desarrollo de la clase.

Al respecto, Silvestre Oramas y Rico Montero, (1997:30) señalan, se concibe el proceso de enseñanza-aprendizaje como un todo integrado, en el que se pone de relieve el papel protagónico del estudiante. En este último enfoque se revela como característica determinante la integración de lo cognitivo y lo afectivo, de lo instructivo y lo educativo, como requisitos psicológicos y pedagógicos esenciales.

Los estudios realizados, por lo general, coinciden varios autores al señalar que las relaciones interdisciplinarias permiten abordar los problemas complejos desde una visión globalizadora, donde las disciplinas, aportan sus núcleos conceptuales y métodos de trabajo, lo que constituye un pensamiento de avanzada en el campo de la didáctica de las ciencias. Ander-Egg (1993:34), Morín (1994:56), Torres Santomé (1994:25), Mañalich (1998:34), Valcárcel (1998:14), Perera (2004:25), Fiallo (2004:16), Salazar (2004:12), Mena (2010:60), entre otros.

Al identificar que en el currículo del Licenciado en Contabilidad y Finanzas hay tres disciplinas Matemática, aporta los procesos lógicos del pensamiento del Contador, Teoría Económica le ofrece los fundamentada científicamente para el análisis de las problemáticas que se gestan durante las relaciones monetarias mercantiles provocadas por la circulación del Capital Financiero y el Derecho la legislación que legaliza dichas operaciones.

El aporte de núcleos conceptuales y métodos de trabajo de estas ciencias al integrarse a la disciplina Contabilidad la convierte desde el punto teórico metodológico en la ciencia que direcciona el proceso de integración horizontal en el año académico y vertical en la carrera al trascender sus fronteras en su enlace con otras materias a fines, estableciendo nexos de cooperación con la Disciplina Principal Integradora durante la realización eficiente de su práctica profesional.

Para lograr que los contenidos curriculares trasciendan en sentido vertical es necesario organizarlos desde un enfoque sistémico integral y centra su atención en la continuidad y secuencia lógica como condición indispensable para garantizar su alcance en espiral, que permita la acumulación de conocimientos en las estructuras o segmentos de la psiquis del sujeto y la creación de nuevas estructuras cognitivas al enfrentarse a nuevas situaciones en el punto de encuentro con otras materias que lo envuelven, por las relaciones que se establecen entre los sujetos con el objeto de estudio durante el proceso de enseñanzaaprendizaje de las ciencias básicas y específicas del año académico y la carrera.

Por otra parte el aprendizaje ha sido abordado a lo largo de la historia desde diferentes tendencias, los cuales han constituido modelos específicos representativos de una determinada teoría. Las más conocidas son: el conductismo, procesamiento de la información, humanismo, constructivismo y la escuela histórico-cultural fundada por Vigotsky. Han aparecido variados trabajos que abordan las diferentes concepciones de aprendizaje, entre los que se encuentran: Pozo (1993:46), Varela (1997:36), Castellanos (2005:34), B. Castellanos (2005:16), Zilberstein y Silvestre (2000:39).

Asumir la concepción histórico-cultural de Vigotsky, al considerar la enseñanza de la Contabilidad en espiral, permitiendo al individuo conformar su personalidad durante el proceso de aprendizaje al realizar actividades que le permitan apropiarse de conocimientos, hábitos, habilidades que regulen su actividad, evidenciando el nivel de desarrollo al considerar como diferencia entre aquello que el sujeto puede realizar con ayuda y lo que es accesible a su actividad independiente, espacio donde la responsabilidad del maestro ampliar la zona de desarrollo próximo de los estudiantes, aprovechando al máximo sus posibilidades de aprendizaje.

Concepción didáctica para la disciplina Contabilidad durante el ciclo básico.

El análisis del objeto y del campo de la investigación revela la necesidad de transformar la concepción didáctica actual para la enseñanza-aprendizaje de la Contabilidad durante el ciclo básico en aras de hacer más 
significativa su incidencia en la formación profesional del Licenciado en Contabilidad y Finanzas. Aunque la Pedagogía, la Didáctica General y la Psicología Educativa han abordado con detenimiento la integración de los contenidos, en el proceso de enseñanzaaprendizaje, la teoría existente presenta limitaciones para introducir en la práctica pedagógica los cambios deseados.

En el presente epígrafe se destacan los fundamentos teóricos que caracterizan el proceso de enseñanza-aprendizaje de la Contabilidad durante el ciclo básico centrado en la integración de los contenidos en la carrera de Contabilidad y Finanzas, a partir de los referentes teóricos asumidos en la investigación y de los resultados del diagnóstico inicial.

El proceso de integración de los contenidos de la disciplina Contabilidad durante el ciclo básico en la formación del Licenciado en Contabilidad y Finanzas.

La fundamentación de la propuesta parte de un concepto alrededor del cual se conciben las principales transformaciones en relación con el objeto de la investigación: el concepto de concepción didáctica para una enseñanzaaprendizaje de la disciplina Contabilidad durante el ciclo básico en la carrera de Contabilidad y Finanzas.

En su conformación se tuvieron en cuenta los criterios dados por Cardona (2007:34), León (2007:18), Frías (2008:45) y Márquez Delgado (2008:30) acerca del término concepción, quienes la definen como una construcción científica, que consiste en la sistematización de regularidades, leyes y principios con el objetivo de describir, comprender, asumir, explicar y contextualizar las representaciones abstractas realizadas sobre el objeto de estudio.
Sin embargo, son los criterios de Valle Lima (2007:64), los que permiten definir con mayor claridad el término en esta investigación, al reconocerla como una categoría ligada a conceptos esenciales que los contiene y explicita los principios que la sustentan, el punto de partida que se asume para la elaboración de las categorías o marco conceptual, así como una caracterización de aquellos aspectos trascendentes que sufren cambios.

Esta interpretación permite reconceptualizar tanto el proceso de integración de los contenidos de la disciplina Contabilidad durante el ciclo básico, como el proceso de enseñanzaaprendizaje que lo sustenta al integrarse con otras ciencias del currículo.

La integración de los contenidos de la Contabilidad con estas ciencias es interpretada como un proceso que promueve las relaciones entre estas disciplinas y la práctica profesional, desconceptualizadas como resultado del desarrollo de procesos de enseñanzaaprendizajes tradicionales. El mismo favorece el redescubrimiento de las propiedades esenciales del objeto de estudio, sus regularidades y nexos con otros contenidos, y su aplicación renovadora a la vida profesional.

Estos razonamientos exigen que cada disciplina esté constituida por un sistema de componentes didácticos integrados para sí, como condición esencial para convertirse en áreas de saberes potencialmente integrables entre sí (hacia las ciencias). El pensamiento anterior permite estructurar a la integración de los contenidos en tres etapas: Campo disciplinar contable, Sistema impreso de medios y el Sistema contable real.

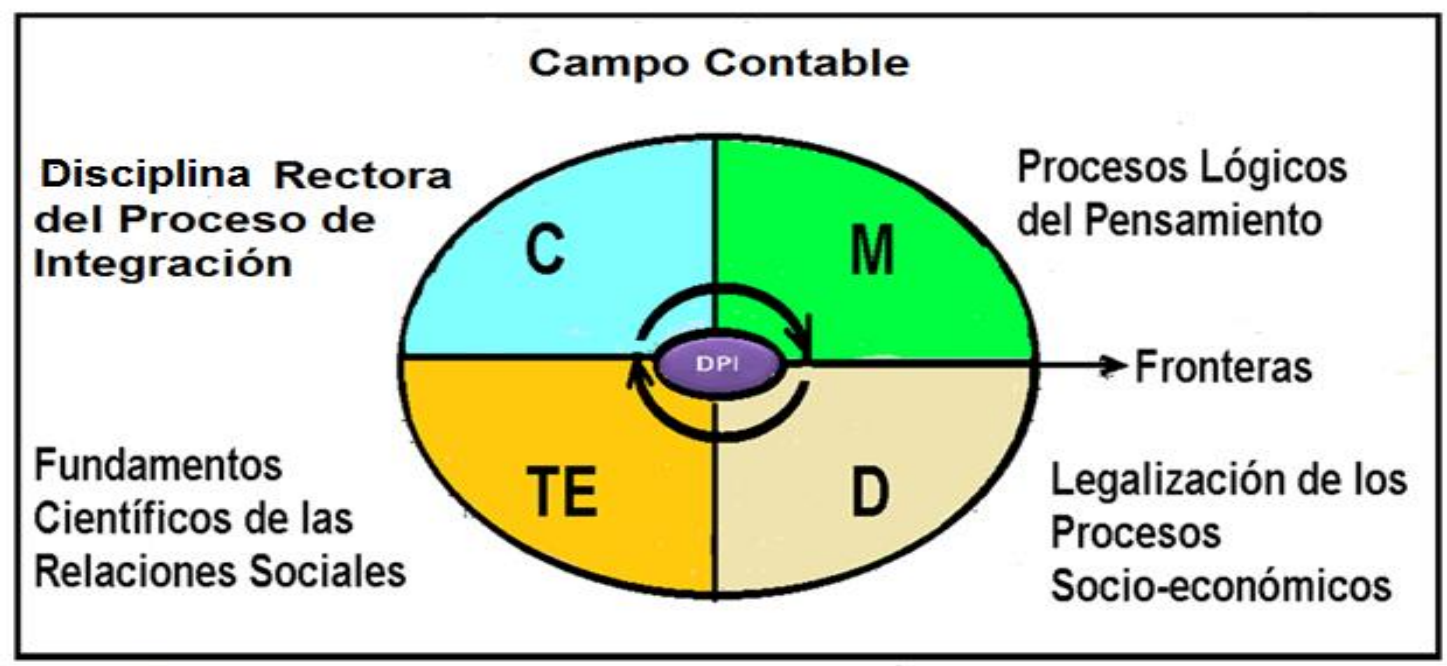

Figura 1.- Estructura cíclica del proceso de integración. 
El campo disciplinar contable en los procesos primarios amplia la visión de los estudiantes más allá de las fronteras de la disciplina de Contabilidad, revelando los puntos de contacto con la disciplina Teoría Económica que fundamenta científicamente los fenómenos sociales y económicos. La disciplina Matemática proporciona los procesos lógicos en la solución de problemas a nivel macro y micro económico y el Derecho legaliza los procesos contables a partir de las transformaciones producidas por relaciones monetarias mercantiles.

Asumir el Modelo propuesto por Silva Palavecinos, (2009:16) modificado del original de Limone (1977:56), no sin antes señalar que lo, referido al Proceso Primario, se observa, según Limone (1977: 9), una relación entre las ¿gráficas? de gastos y de caja. Este modelo pretende facilitar el aprendizaje integrado de las disciplinas de Administración, Finanzas y Contabilidad. Sin embargo, su contribución es limitada pues fija la idea de que gasto es sinónimo de desembolso, es decir, de salida de dinero. En aras de su perfeccionamiento, se propuso un nuevo modelo, que no logra incluir a las disciplinas Matemática, Teoría Económica, Derecho y las disciplinas de Formación General que lo transversalizan. Por lo que se propone un nuevo modelo Campo disciplinar contable en los procesos primarios.

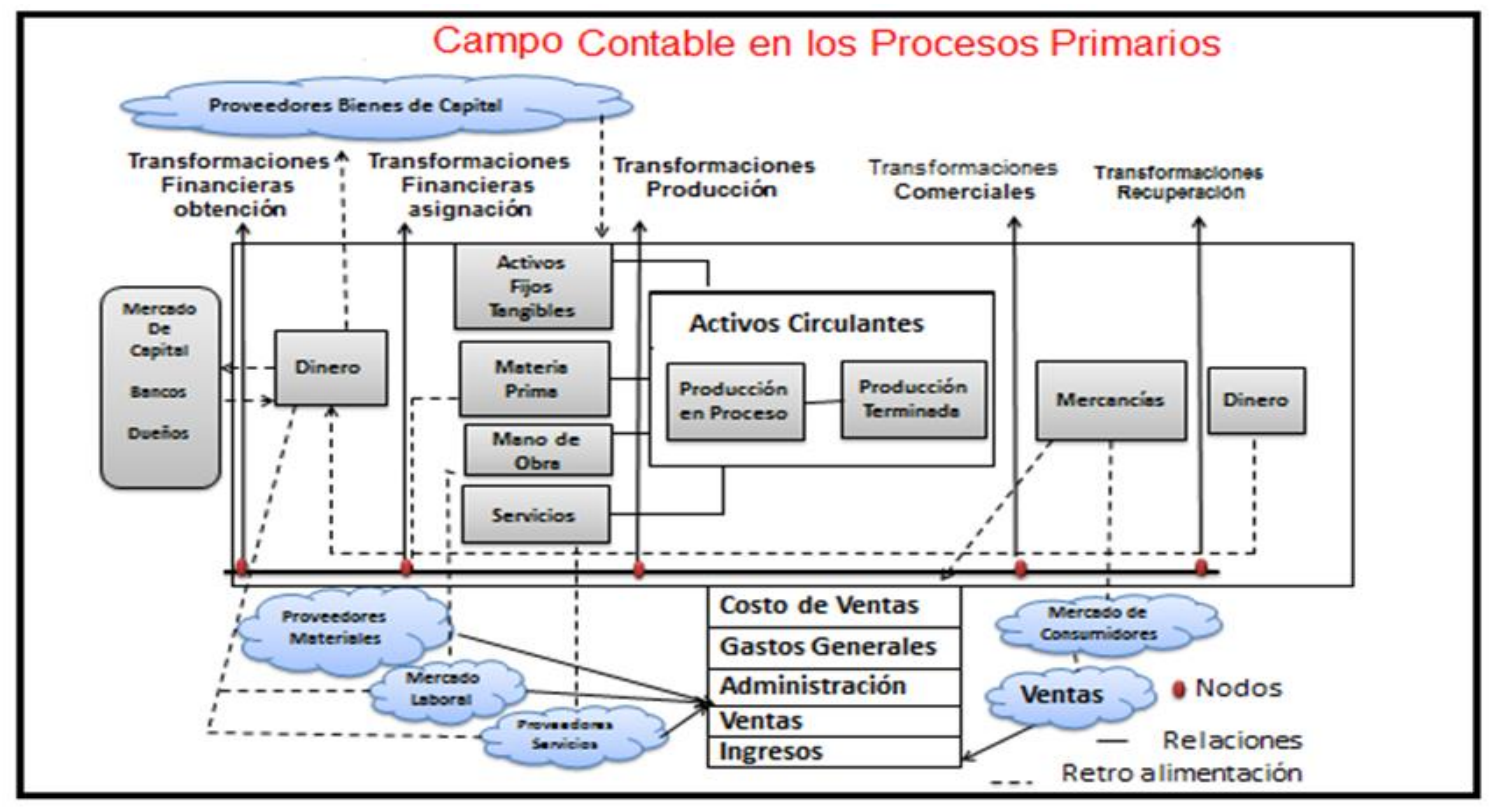

Figura 2.- Adaptación propia del modelo de Berta Silva Palavecinos (2009).

En él se podrá observar que se ha incorporado un nuevo elemento necesario para la enseñanza interdisciplinar, Campo Disciplinar Contable en los Procesos Primarios, (nodos) punto de encuentro entre las disciplinas del currículo.

En esta etapa de la integración los estudiantes reconocen con mayor claridad la necesidad de trabajar en equipos, diseñados por subsistemas contables y un sistema de medios impresos que permiten el control y funcionamiento del sistema contable, aplicando lo aprendido en el campo anterior, desde una visión distinta a la fragmentación.

En esta etapa se diseña una empresa simulada que modele los procesos contables donde fluyen y se transforman recursos, materiales e inmateriales, de manera iterativa. En este ciclo el estudiante experimenta las transformaciones que sufren los recursos por el consumo y utilización para mantener la estructura y el funcionamiento de la empresa, los que deben recuperarse a través del desarrollo del mismo y mientras la empresa continúe su ciclo termina donde comienza. (Palavecinos, 2009:8)

Este ciclo puede durar más o menos un período de tiempo predefinido y utilizado para diagnosticar el desempeño de los sujetos que participan durante las transformaciones del objeto de estudio. Provocadas por la integración del campo contable y el sistema de medios impresos durante proceso de modelación de la empresa simulada tributando en espiral a la disciplina principal integradora de forma horizontal en el año académico y vertical en la carrera.

Al respecto Berta Silva Palavecinos (2009:16), generalmente este se repite muchas veces durante un mismo período de tiempo, además, se podría afirmar que el sistema se 
retroalimenta a sí mismo, se auto mantiene y mientras esto ocurra puede permanecer indefinidamente en funcionamiento. Semestre, un año como lo planifique el profesor o el colectivo de año.

Las reflexiones anteriores conducen al autor a asumir una primera relación esencial proceso de integración ciencias básicas, básicas específicas de la profesión y la disciplina principal integradora.

La relación entre campo disciplinar contable de los procesos primarios, sistema de medios y el sistema contable, disciplina principal integradora.

El tránsito gradual de la integración de estos tres momentos con la disciplina principal integradora, se logra, a partir de la utilización de un sistema de ejercicios integrales que le permite al estudiante de forma organizada rotar por los diferentes subsistemas contables (Caja Banco, Inventarios, Activos Fijos Tangibles, Cobros y Pagos, Costo), interactuando con los documentos primarios (Factura, Conduce, Informe de Recepción, Recibo de Ingreso, Cheque, Letras de Cambio, otros), libros básicos (Libro Diario y Libro Mayor) y submayores de (Inventario, Cuentas por Cobrar y Pagar, Elementos de Gasto, otros).

En la solución de las problemáticas provocadas por las relaciones monetarias mercantiles que se establecen en el proceso de producción y prestación de servicios logrando concientizar y estimular el desempeño profesional de los que participan en el proceso de enseñanzaaprendizaje, finalizando cada período con el Estado de Balance General iniciando el próximo con los resultados obtenidos del anterior.

Este proceso ciclo lleva implícito un alto nivel de integración y sistematización que permite al estudiante apropiarse del algoritmo de trabajo de un profesional de las ciencias contables, pasando del dominio de los contenidos disciplinares adquiridos, a su aplicación en la solución de los problemas, la formación de habilidades y capacidades para perfeccionar e implementar un sistema contable en cualquier tipo de empresa. Esta conclusión nos arriba a la segunda relación esencial.

\section{La relación entre la Universidad-Empresa.}

El diseño de una empresa simulada como forma de organización del proceso de enseñanza-aprendizaje de la disciplina Contabilidad, exige una relación armónica entre los componentes personales y no personales, con todos los actores de la actividad empresarial.

\section{Componentes esenciales que estructuran la concepción didáctica.}

La concepción didáctica asume las relaciones que se establecen en el campo interdisciplinar contable en los procesos primarios. Lo que significa el mantenimiento estable de las relaciones esenciales como condición para la materialización de las regularidades de la concepción que se propone.

En su identificación se parte de la definición dada por Sierra (1995:32), quien considera que la regularidad "expresa un cierto grado de obligatoriedad en las relaciones del carácter causal, necesaria y estable entre los fenómenos y propiedades del mundo objetivo, lo que implica que un cambio de algún aspecto exige la transformación de otro". Desde esta perspectiva, y la de asumir al proceso de integración de los contenidos en su tránsito gradual desde lo disciplinar, a lo interdisciplinar y de este campo interdisciplinar contable en los procesos primarios, surge la primera regularidad de la concepción didáctica, cuya esencia está dada en:

A través de esta regularidad se materializa el carácter sistémico de los componentes didácticos no personales del proceso de enseñanza-aprendizaje de la Contabilidad durante el ciclo básico, centrado en la integración de los contenidos. En la misma se regulan los vínculos entre la Matemática, Teoría Económica y el Derecho, y de estas con la (DPI).

La segunda regularidad es el resultado de concebir a este proceso armónico y totalizador desde la sistematización de la enseñanza y del aprendizaje. Su esencia está dada en:

La relación entre la dirección integrada del aprendizaje y la apropiación integrada de los contenidos de las ciencias básicas y específicas.

Estas regularidades, manifestadas en forma estable, permiten fundamentar un sistema de principios cuyas funciones, lógicagnoseológica y práctica, rigen el proceso de enseñanza-aprendizaje estudiado.

Estas regularidades, manifestadas en forma estable, permiten fundamentar un sistema de principios cuyas funciones, lógicagnoseológica y práctica, rigen el proceso de enseñanza-aprendizaje estudiado.

En la identificación de los mismos se tuvo en cuenta el sistema de principios que dinamizan al proceso de enseñanza-aprendizaje desarrollador, fundamentado desde la Didáctica Integradora (Silvestre y Zilberstein, 
2000:32), y que tiene sus bases teóricas en el Enfoque Histórico-Cultural de Vigotsky y sus seguidores.

De esta manera, se conciben los siguientes: Principios Dinamizadores.

$\checkmark$ Principio del carácter cíclico en espiral del proceso de enseñanza-aprendizaje de la Contabilidad durante el ciclo básico.

$\checkmark$ Principio del carácter rector de las relaciones interdisciplinarias en el proceso de enseñanza-aprendizaje de la disciplina Contabilidad durante el ciclo básico.

$\checkmark$ Principio del carácter mediador del colectivo pedagógico integrado.

$\checkmark$ Principio del carácter activo de los estudiantes durante la apropiación integrada de los contenidos de las ciencias básicas y específicas de la profesión.

$\checkmark$ Principio del carácter integrador de la relación universidad-empresa.

De esta manera, el proceso de integración de los contenidos se estructura teniendo en cuenta las regularidades esenciales y principios que armonizan la concepción didáctica y fundamentan su estructura en etapas desarrolladoras.

\section{PRIMERA ETAPA: Diagnóstico.}

Comprende las primeras seis semanas del curso y constituye la etapa de preparación de los estudiantes. La puesta en práctica de la concepción se inicia en la Unidad Didáctica No 2: Registros Básicos de la Contabilidad, donde se desarrolla un diagnóstico que brinda la información acerca del estado inicial de las variables que condicionan el objeto de estudio.

Objetivo: Diagnosticar el estado actual de los estudiantes que ingresan a la carrera de Contabilidad y Finanzas desde el punto de vista cognitivo, afectivo y actitudinal en las ciencias básicas y las de la profesión.

SEGUNDA ETAPA: Proceso de integración de los contenidos.

Objetivo: Adiestrar a los estudiantes en el uso de los medios y objetos de trabajo que utilizan los profesional de las ciencias contables, en la solución de ejercicios integrales vinculados a la vida real empresarial, donde están presentes la Matemática, Teoría Económica y Práctica Profesional del Contador.

Esta etapa se caracteriza por los siguientes indicadores:

Diseño de la empresa simulada que modela los subsistemas contables de forma integrada a los medios y objetos de trabajo del contador en la solución de los ejercicios integradores vinculados a la práctica profesional.

$\checkmark$ Seguimiento sistemático al desarrollo potencial de los estudiantes.

$\checkmark$ Familiarización con el aprendizaje colaborativo y con el trabajo en equipos de contadores (según el rol que desempeñan, Contador Principal o Contadores Auxiliares).

$\checkmark$ Determinación de los núcleos básicos interdisciplinarios entre las ciencias básicas y la profesión al reconocer a la Contabilidad como la ciencia que se nutre de los resultados del análisis de otras ciencias y su traducción a un lenguaje universal.

TERCERA ETAPA: Desarrollo del proceso de integración de los contenidos.

Abarca el segundo semestre del primer año y primer semestre de segundo año y constituye la etapa donde los estudiantes desarrollan sus habilidades investigativas integradoras en la resolución de problemas integradores de mayor complejidad en la práctica profesional.

Objetivo: Consolidar en los estudiantes el uso del método investigativo y del algoritmo de trabajo del profesional contable durante la solución de problemas integradores provenientes de las empresas del territorio donde están presentes las ciencias básicas, específicas y las de la profesión.

Esta etapa se caracteriza por los siguientes indicadores:

$\checkmark$ Mantenimiento del control diagnóstico a la apropiación integrada de los contenidos.

$\checkmark$ Determinación de los núcleos básicos interdisciplinarios y básico-profesionales.

$\checkmark$ Diseño de talleres integradores en el colectivo de año que promuevan la presencia (según los objetivos de la actividad) de los profesores y tutores en las empresas.

$\checkmark$ Planificación de auditorías por el colectivo de la disciplina Contabilidad y Auditoría para auditar los equipos de trabajo en la empresa simulada y así conocer el desarrollo del estado actual y final de las necesidades profesionales del equipo evaluado.

En consecuencia, el proceso de integración de los contenidos de las ciencias básicas puede ser estructurado atendiendo a los progresos formativos de los estudiantes y a los niveles de intervención y mediación de colectivo 
pedagógico integrado. Estos procesos inciden directamente en la apropiación integrada de los contenidos de las ciencias básicas y específicas de la profesión repercuten en la formación de un mejor desempeño del Licenciado en Contabilidad y Finanzas.

La relación dialéctica y esencial que se establece entre las regularidades y los principios de la concepción didáctica permite estructurar el proceso de integración de los contenidos de las ciencias básicas y específicas de la profesión en etapas, las que armonizan atendiendo al carácter desarrollador del proceso de enseñanza-aprendizaje de la Contabilidad durante el ciclo básico que sustenta y se estructura como resultado del carácter sistémico de sus componentes didácticos. Dicha trascendencia es posible, asumiendo el carácter rector de la disciplina Contabilidad y el rol del profesor y los estudiantes en su desempeño profesional.

\section{Conclusiones}

$\checkmark$ Los referentes teóricos asumidos en la investigación permitieron fundamentar una concepción didáctica del proceso de enseñanza-aprendizaje de la Contabilidad durante el ciclo básico, centrado en la integración de los contenidos.

$\checkmark$ El proceso de integración de los contenidos de las ciencias básicas trasciende a los componentes didácticos del proceso de enseñanza-aprendizaje que lo sustenta, entendiendo a este como la vía profesionalizada, de carácter sistémico, activador y mediatizado, que conduce a los estudiantes de Contabilidad y Finanzas a la apropiación integrada de los contenidos, como resultado de la dirección integrada del aprendizaje, por el colectivo pedagógico del año integrado.

$\checkmark$ La concepción didáctica propuesta es dinamizada por un sistema de principios que regulan el alcance del proceso de integración de los contenidos de las ciencias básicas y específicas de la profesión, estructurándolo en etapas secuenciadas (diagnóstico, acercamiento, desarrollo y consolidación) que favorecen la formación básica y profesional del Licenciado en Contabilidad y Finanzas, desde el proceso de enseñanza-aprendizaje de la Contabilidad durante el ciclo básico.

\section{Referencias}

Adler y Milne, (1997). En: http://www.ifac.org/system/files/newfiles/enewIAESB

Ander-Egg, E. (1993). Interdisciplinariedad en educación. Buenos Aires: Magisterio del Río de la Plata.

Cardona, G. (2007). Concepción Pedagógica del Proceso de Formación Laboral para estudiantes de Tecnología en Comercio Internacional de ESUMER (Medellín, Colombia). Tesis Doctoral. Pinar del Río: CECES.

Castellanos, D y otros. (2005). Aprender y enseñar en la escuela. La Habana: Pueblo y Educación.

Castellanos, D y otros. (2005). Aprender y enseñar en la escuela. La Habana: Pueblo y Educación.

Donoso A NESJ: (2009) Dirección "La docencia de la Contabilidad a debate " Ed. Kronos, Sevilla

Donoso A Y López., (2010). Dirección "La docencia de la Contabilidad a debate " Ed. Kronos, Sevilla.

Fiallo, J. (2001). La interdisciplinariedad en la escuela: de la utopía a la realidad. Curso Prereunión Pedagogía 2001. La Habana. En formato digital.

Frías, Y. (2008). Concepción didáctica del proceso de enseñanza-aprendizaje semipresencial en la Universidad de Pinar del Río. Tesis Doctoral. Pinar del Río: CECES.

Hwang et al., (2005) En: www/htpp. American Accounting Association (AAA)

Konja, J. V. (2008). Plan de Estudio para la Formación del Contador Público en América Latina.

León, V.E. (2007). Una concepción didáctica para la profesionalización del proceso de enseñanza-aprendizaje de la física en la formación del bachiller técnico en agronomía. Tesis Doctoral. Pinar del Río: ISP.

Limone, Aquiles. (1977)L'autopoiese dans les organisations. Tesis doctoral, Université Paris IX Dauphine, Paris, Francia, 1977.

Mañalich, R. (1998). Interdisciplinariedad y didáctica: vías para la transformación del desempeño profesional de los docentes de humanidades. Taller interdisciplinar con jefes de departamento de humanidades de los 
centros de referencia de Ciudad de La Habana. Material impreso. La Habana: MINED.

Márquez Delgado, D.L. (2008). Concepción pedagógica del proceso de formación de los estudiantes de la carrera de Estudios Socioculturales a través del modo de actuación: Estrategia para su implementación en la Universidad de Pinar del Río. Tesis Doctoral. Pinar del Río: CECES.

Mena, J.L. y otros. (2010). Estudio diagnóstico acerca del desarrollo de las habilidades de comprensión en estudiantes de ciencias de universidades iberoamericanas. Libro de Actas del VI Congreso Internacional de Didáctica de las Ciencias y XI Taller Internacional sobre la Enseñanza de la Física. Marzo 2010, s/p. ISBN 978-959-18-0541-6.

Ministerio de Educación Superior (MES). Reglamento para el Trabajo Docente y Metodológico en la Educación Superior. La Habana, Cuba. 2007.

Moracho, F. S. (1915). Formación de Auditores. Edit. Esparta. España.

Morín, E. (1994). Introducción al pensamiento complejo. Barcelona: Gedisa.

Perera, F., (2000) Enfoque interdisciplinar profesional de la enseñanza aprendizaje de la Física para la especialidad de Biología de la Licenciatura en Educación. Tesis presentada en opción al grado científico de Doctor en Ciencias Pedagógicas. Ciudad de la Habana, Instituto Superior Pedagógico Enrique José Varona.

Pozo, J.I. (1993). ¿Por qué los alumnos no aprenden la ciencia que les enseñamos?: El caso de las ciencias de la tierra. Enseñanza de las Ciencias de la Tierra, 8 (1).

Sacristán (1992). Hacia un currículo global. Edit. Morata, España.
Salazar, D. (2001). La formación interdisciplinaria del futuro profesor de Biología en la actividad científico-investigativa. Tesis de Doctorado. La Habana: ISPEJV. En formato digital.

Salazar, J. (2004). Prospectiva Tecnológica y consideraciones curriculares en la ingeniería agrícola. Colombia: Universidad Nacional.

Sierra Salcedo, R. (1997). Estrategias y alternativas pedagógicas. Tesis de Maestría. ISPEJV. Ciudad de la Habana. En formato digital, doc.

Silva Palavecinos, B. (2009) "El aprendizaje de los fundamentos contables basado en el enfoque del Proceso Primario".

Silvestre, M. y Rico, P. (1997). El proceso de enseñanza aprendizaje. La Habana: ICCP.

Silvestre, M. y Zilberstein, J. (2000). Enseñanza aprendizaje desarrollador. España: Morata.

Torres Santomé, J. (1994). Globalización e interdisciplinariedad: el currículum integrado. Madrid: Morata.

Tristá, B. (1985). La Educación Superior en Cuba. CEPES. Universidad de la Habana, Cuba.

Valcárcel, R. (1998). Estrategia interdisciplinaria de superación para profesores de ciencia. Tesis Doctoral: ISPEJV. En formato digital.

Valera, O. (1997). Problemas actuales de la Pedagogía y la Psicología pedagógica. Formato digital. La Habana.

Valle Lima, A.D. (2007). Metamodelos de la Investigación Pedagógica. La Habana: ICCP. En formato digital. 